\title{
Nonalcoholic Lipid Accumulation and Hepatocyte Malignant Transformation
}

\author{
Juanjuan $\mathrm{Gu}^{\# 1}$, Min Yao ${ }^{\# 2}$, Dengbing Yao ${ }^{3}$, Li Wang ${ }^{4}$, Xuli Yang ${ }^{1}$ and Dengfu Yao*1 \\ ${ }^{1}$ Research Center of Clinical Medicine and Department of Oncology, Affiliated Hospital of Nantong University, Nantong, \\ Jiangsu, China; ${ }^{2}$ Department of Immunology, Medical School of Nantong University, Nantong, Jiangsu, China; \\ ${ }^{3}$ School of Life Sciences, Nantong University, Nantong, Jiangsu, China; ${ }^{4}$ Department of Medical Informatics, \\ Medical School of Nantong University, Nantong, Jiangsu, China
}

\begin{abstract}
Worldwide incidence of hepatocellular carcinoma (HCC) is steadily increasing, highlighting its status as a public health concern, particularly due to its significant association with other comorbidities, such as diabetes. However, nonalcoholic fatty liver disease (NAFLD) has emerged as a primary risk factor, with its own prevalence increasing in recent years, and it has gradually caught up with the historical primary etiological factors of infection with hepatitis B virus and hepatitis $C$ virus, exposure to aflatoxin, or alcohol liver disease. The deeply worrisome aspects of all of these high risk factors, however, are their remarkable presence within populations. Systemic and genetic mechanisms involved in the malignant transformation of liver cells, as well as useful biomarkers of early stage HCC are being investigated. However, the exact mechanisms underlying the interrelation of NAFLD and HCC remain largely unknown. In this review, some of the recent advances in our understanding of liver lipid accumulation are summarized and discussed to provide insights into the relationship between NAFLD and hepatocyte malignant transformation. (C) 2016 The Second Affiliated Hospital of Chongqing Medical University. Published by XIA \& HE Publishing Inc. All rights reserved.
\end{abstract}

\section{Introduction}

Hepatocellular carcinoma (HCC) is the $5^{\text {th }}$ most common malignant tumor in the world, ranking as the $3^{\text {rd }}$ most frequent cause of cancer mortality. ${ }^{1,2}$ In China alone, HCC ranks as $2^{\text {nd }}$

Keywords: Nonalcoholic fatty liver disease; Hepatocellular carcinoma; Metabolism.

Abbreviations: $\mathrm{CPT}$, carnitine palmitoyltransferase; ER, endoplasmic reticulum; FFA, free fatty acid; ERK, extracellular signal-regulated kinase; GWAS, genomewide association studies; HBV, hepatitis B virus; HCC, hepatocellular carcinoma; $\mathrm{HCV}$, hepatitis C virus; HSC, hepatic stellate cell; IL-6, interleukin-6; IR, insulin resistance; miR, microRNA; NAFLD, nonalcoholic fatty liver disease; NASH, nonalcoholic fatty hepatitis; NF-кB, nuclear factor kappa B; NKT, natural killer T cells; OPN, osteopontin; PNPLA, patatin-like phospholipase domain-containing protein; RNS, reactive nitrogen species; ROS, reactive oxygen species; SNP, single nucleotide polymorphism; SREBP, sterol regulatory element binding protein; TAGE, toxic advanced glycation end-products.

Received: 17 March 2016; Revised: 13 May 2016; Accepted: 14 May 2016

DOI: $10.14218 / \mathrm{JCTH} .2016 .00010$.

\#These authors contributed equally to this work

*Correspondence to: Professor Dengfu Yao, Research Center of Clinical Medicine, Affiliated Hospital of Nantong University, Nantong 226001, Jiangsu Province, China Tel: +86-513-85052297, Fax: +86-513-85052554, E-mail: yaodf@ahnmc.com among all malignancies, with its mortality rate being almost equal to its morbidity rate; moreover, a large portion of cases has been localized to the inshore area of the Yangtze River. The development of HCC is a complex multi-step process involving multiple genes. Major risk factors of HCC include hepatitis $B$ virus (HBV) or hepatitis $C$ virus (HCV) infection, alcoholic liver disease, and exposure to nitrosamines, aflatoxin, and other harmful substances. ${ }^{3}$ Chronic persistent infection with hepatoviruses remains the main pathological factor of inducing cirrhosis and HCC in nonalcoholic individuals in China; however, the recent decades have seen a sweeping change in the Chinese dietary structure and lifestyle, and this has been accompanied by a drastic increase in incidence of fatty liver disease (FLD). ${ }^{4}$

The median prevalences of alcoholic fatty liver disease (AFLD) and nonalcoholic fatty liver disease (NAFLD) have been reported as $4.5 \%$ and $15.0 \%$, respectively. ${ }^{5}$ Unfortunately, when left untreated, both nonalcoholic steato-hepatitis (NASH) and alcoholic hepatitis can progress to liver fibrosis, cirrhosis and liver cancer; although, the exact underlying mechanisms that lead to malignant transformation of infected liver cells remain largely unknown. Most HCC patients die quickly because of rapid tumor growth, and surgical operation or liver transplantation remains the only effective treatment available for $\mathrm{HCC}^{4}{ }^{4}$ This review summarizes the latest advances in our collective understanding of the relationship between NAFLD and hepatocyte malignant transformation.

\section{Mitochondria and fatty $\beta$-oxidation}

\section{Mitochondria}

The liver is one of the most important organs for maintaining energy supply and carrying out lipid metabolism in the human body. The peroxisomal compartment in hepatocytes host several essential metabolic conversions. Such conversions, however, are defective in peroxisomal disorders, as a result of either failure to properly or adequately import enzymes into the organelle or presence of mutations in the enzymes themselves or their cognate transporters that are necessary to transfer the substrates across the peroxisomal membrane (Fig. 1). Hepatic pathology is one of the cardinal features in disorders of peroxisome biogenesis and peroxisomal $\beta$-oxidation, although it only rarely determines clinical fate. Besides these morphological changes, however, the peroxisome malfunctions cause other observable impacts 


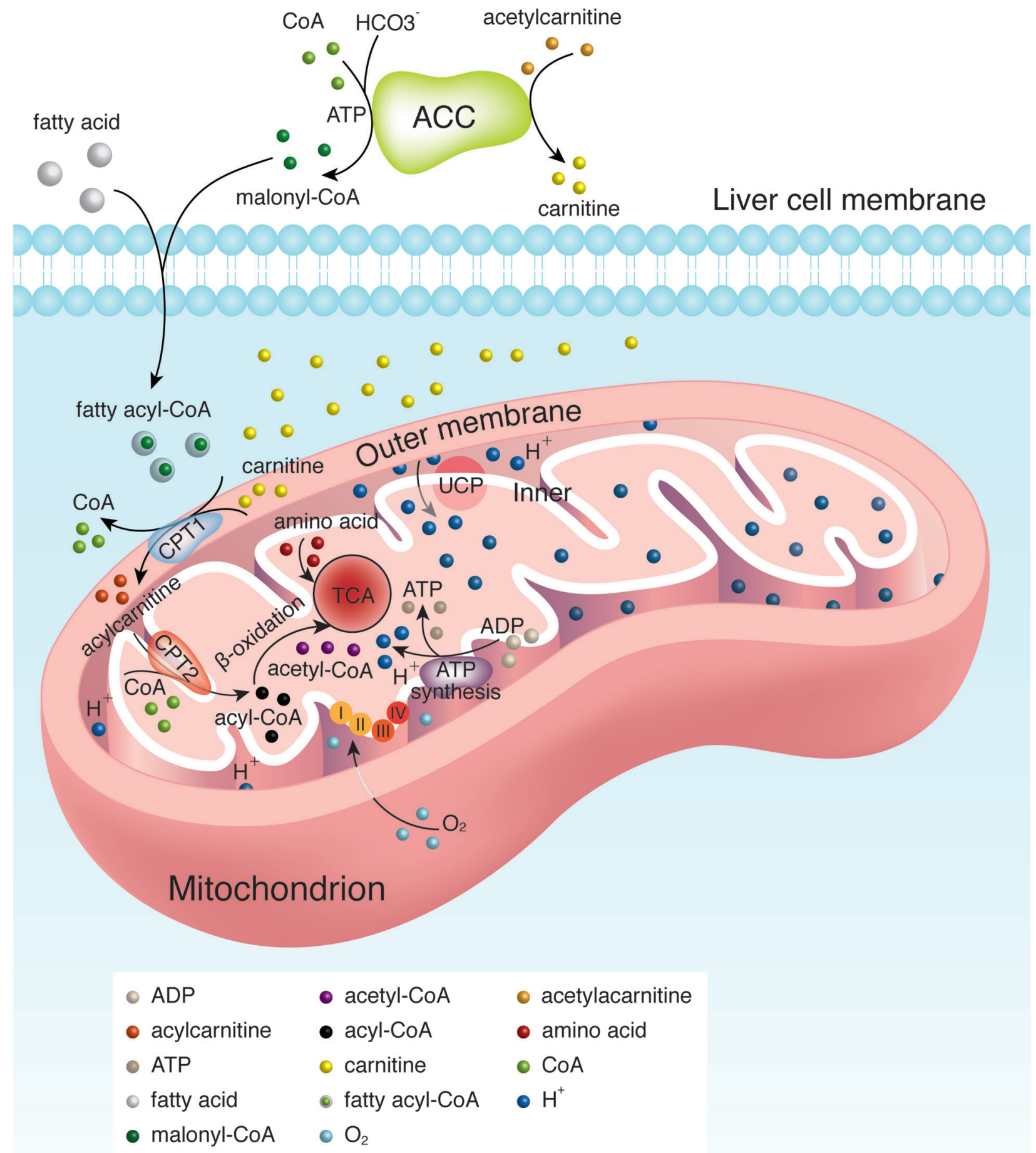

Fig. 1. Abnormality of fatty acid oxidation in mitochondria. Carnitine palmitoyltransferase (CPT)-I or CPT-II regulate fatty acid metabolism. Mitochondrial fatty acid $\beta$-oxidation requires successive carnitine acyltransferases to translocate acyl-coenzyme A (acyl-CoA) from the cytoplasm into the mitochondrial matrix. CPT-I, as an initial and rate-limiting enzyme, generates acyl-carnitines that traverse mitochondrial membranes via specific transporters to enter the mitochondrial matrix. Then, CPT-II produces acyl-COA from acyl-carnitine for fatty acid $\beta$-oxidation to acetyl-COA, after which carnitine crosses the mitochondrial inner membrane and binds to the endogenous or exogenous acyl-CoA to prevent accumulation of acyl-COA, causing cell poisoning.

Abbreviations: CoA, Coenzyme A; ACC, Acetyl-CoA carboxylase; TCA, Tricarboxylic acid cycle; UCP, Uncoupling protein; I II III IV, Electron transfer complex. 
on various cellular compartments, such as the mitochondria and endoplasmic reticulum (ER). ${ }^{6}$

\section{Carnitine level}

Carnitine is a physiological substance that is essential for the proper metabolism of fat and energy production; it serves in transport of both long and medium fatty acid chains. Carnitine attracts these fatty acid chains, breaks them down and then carries them to the mitochondria of the cells where they are metabolized (burned). The ultimate result is that the body burns more fats, and in the process, provides the body with more natural energy. Previous studies have revealed that the circulating carnitine analogue 3-(2, 2, 2-trimethyl hydrazinium) propionate dihydrate (THP) affects increased liver weight due to lipid accumulation in wild-type mice (Fig. 2). ${ }^{7,8}$

\section{Carnitine palmitoyltransferases (CPTs)}

Hepatic CPT-II is a mitochondrial protein that is transported to the mitochondrial inner membrane. It, together with CPT-I, oxidizes long-chain fatty acids in mitochondria. Mutation of its encoding gene or defects in the protein product are associated with mitochondrial long-chain fatty acid oxidation disorders. Decreased activity of CPTs is a hallmark of disorders of mitochondrial fatty acid oxidation with autosomal recessive modes of inheritance. The CPT variants exert a dominantnegative effect on the homo-tetrameric protein of the enzyme (Fig. 3), featuring reduced activities, thermal instability, decreased fatty acid $\beta$-oxidation $(30 \sim 59 \%)$, decreased intracellular ATP (48 79\%), marked reduction of mitochondrial membrane potential at $41^{\circ} \mathrm{C}$, and shortened half-lives; moreover, these variant proteins are polyubiquitinated and rapidly degraded by a lactacystin-sensitive proteasome pathway. ${ }^{6}$ The unstable CPT-II variants, with decreased enzymatic activities, may cause mitochondrial fuel utilization to fall below the phenotypic threshold during conditions of high fever, and thus might represent potential mechanisms for NAFLD.

\section{Abnormal liver lipid accumulation}

\section{Lipid accumulation}

Lipid accumulation in liver or HCC will cause alterations in tumor-associated molecular signaling, including pathways involving the common factors of nuclear factor-kappa $B$ (NF-кB), c-Jun N-terminal kinase (JNK) activation protein-1, as well as overexpression HCC-related genes. For example, hepatic unsaturated fatty acids inhibit the expression of phosphatase and tensin homologue (PTEN) via activation of an NF-кB/mammalian target of rapamycin (mTOR) complex. ${ }^{9}$ PTEN is a tumor suppressor gene, which regulates the PKB/ serine-threonine kinase protein kinase $B$ (akt) pathway, and its deficiency induces liver cell proliferation, thereby inhibiting cell apoptosis and promoting tumor formation; these processes have been confirmed in mouse models of liver-specific PTEN deficiency that show a phenotype resembling NASH features of human, with development of liver steatosis, hepatocyte inflammation and/or fibrosis. ${ }^{10}$

DNA damage can affect hepatic lipid metabolism as well. Reactive oxygen species (ROS) are known to be an important factor in carcinogenesis. ROS have been shown as increased in NAFLD patients with contiguous DNA damage; their induction
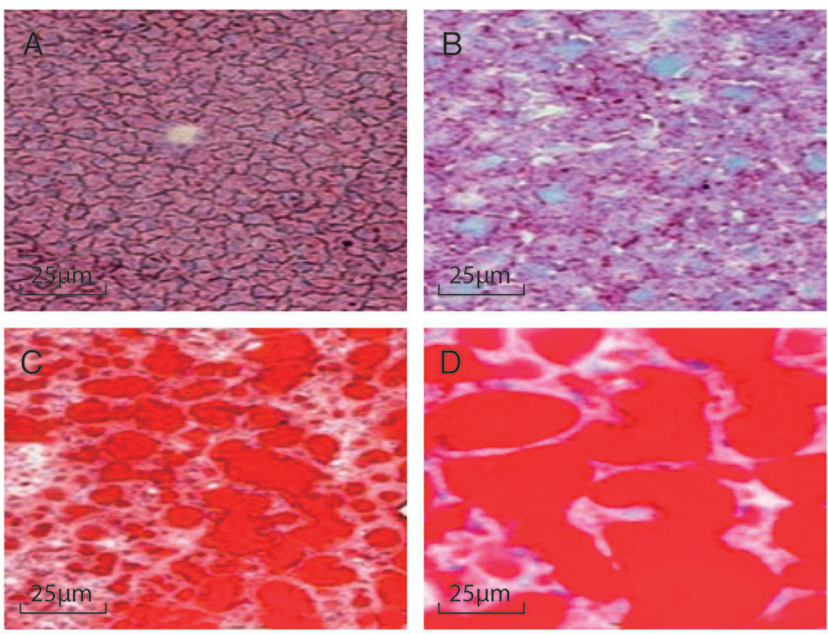

E

Oil Red O Staining-Liver

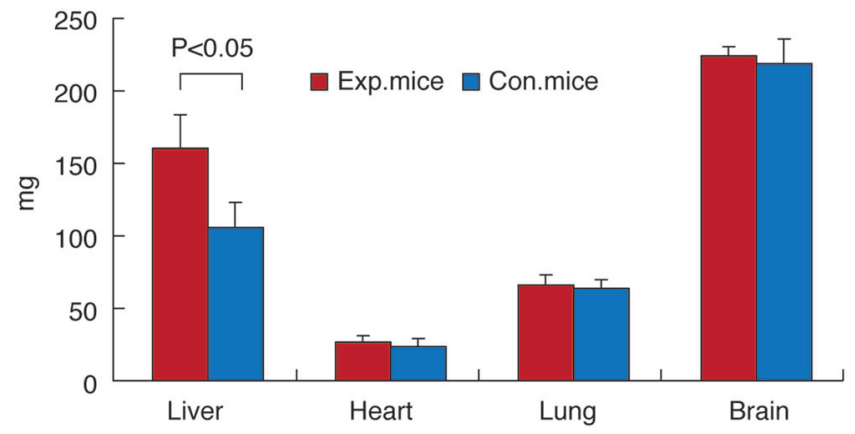

Fig. 2. Hepatic lipid accumulation with carnitine analogues treatment. A-D. Oil red O staining of liver lipids. A \& B. Liver tissues of control mice. C \& D. Liver tissues of experimental mice. E. Weight changes in different tissues of mice treated with carnitine analogues.

can be mediated by hepatic inflammatory cytokines or hepatitis virus infection and reactions with polyunsaturated fatty acids derived from hepatocyte membrane phospholipids that result in production of reactive aldehydes as lipid oxidation (LPO) byproducts. One of such byproducts, 4-hydroxynonenal (4 HNE), can react with DNA to form mutagenic exocyclic etheno-DNA adducts, preferentially forming in codon 249 of TP53 and resulting in inactivation of the tumor suppressor p53 and providing a secondary growth advantage and cellular resistance to apoptosis. ${ }^{11}$

\section{Adipokines}

Accumulating basic and clinical data have demonstrated that leptin plays an important role in hepatic stellate cell (HSC) activation and hepatic fibrosis, in many aspects of both. First, leptin can stimulate production of the tissue inhibitor of metalloproteinase-1 (TIMP-1) via the Janus kinase/signal transducer and activator of transcription (JAK/STAT) pathway, thereby promoting fibrogenesis. Second, it can act on hepatic endothelial cells, thereby promoting tube formation and migration and affecting angiogenesis. ${ }^{12}$ Finally, the level of leptin expression is significantly higher in NAFLD patients, and it has been 
Gu J. et al: NAFLD in HCC

A

CPT-II Gene

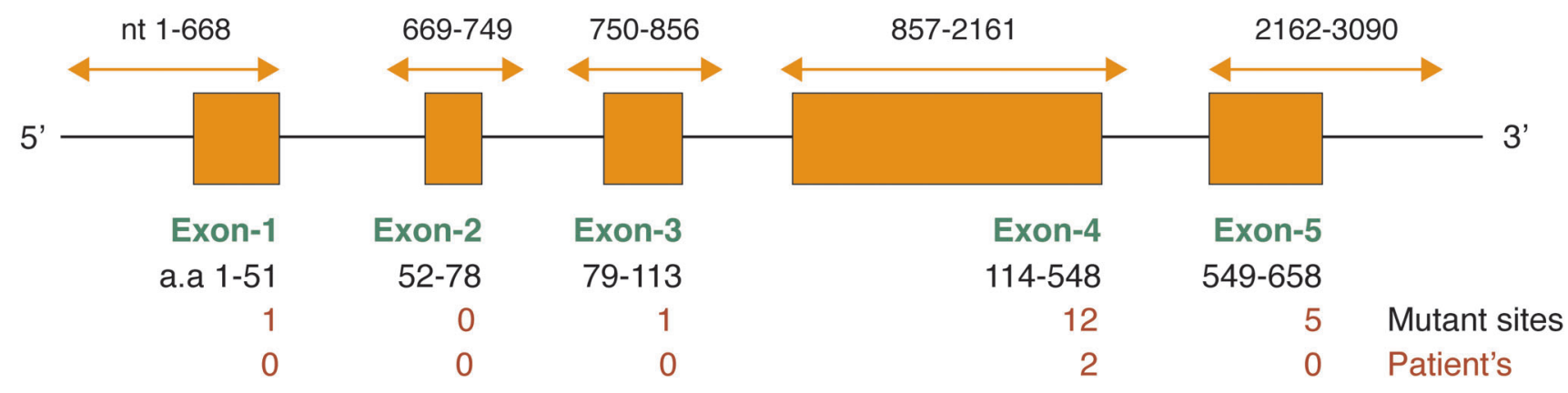

B

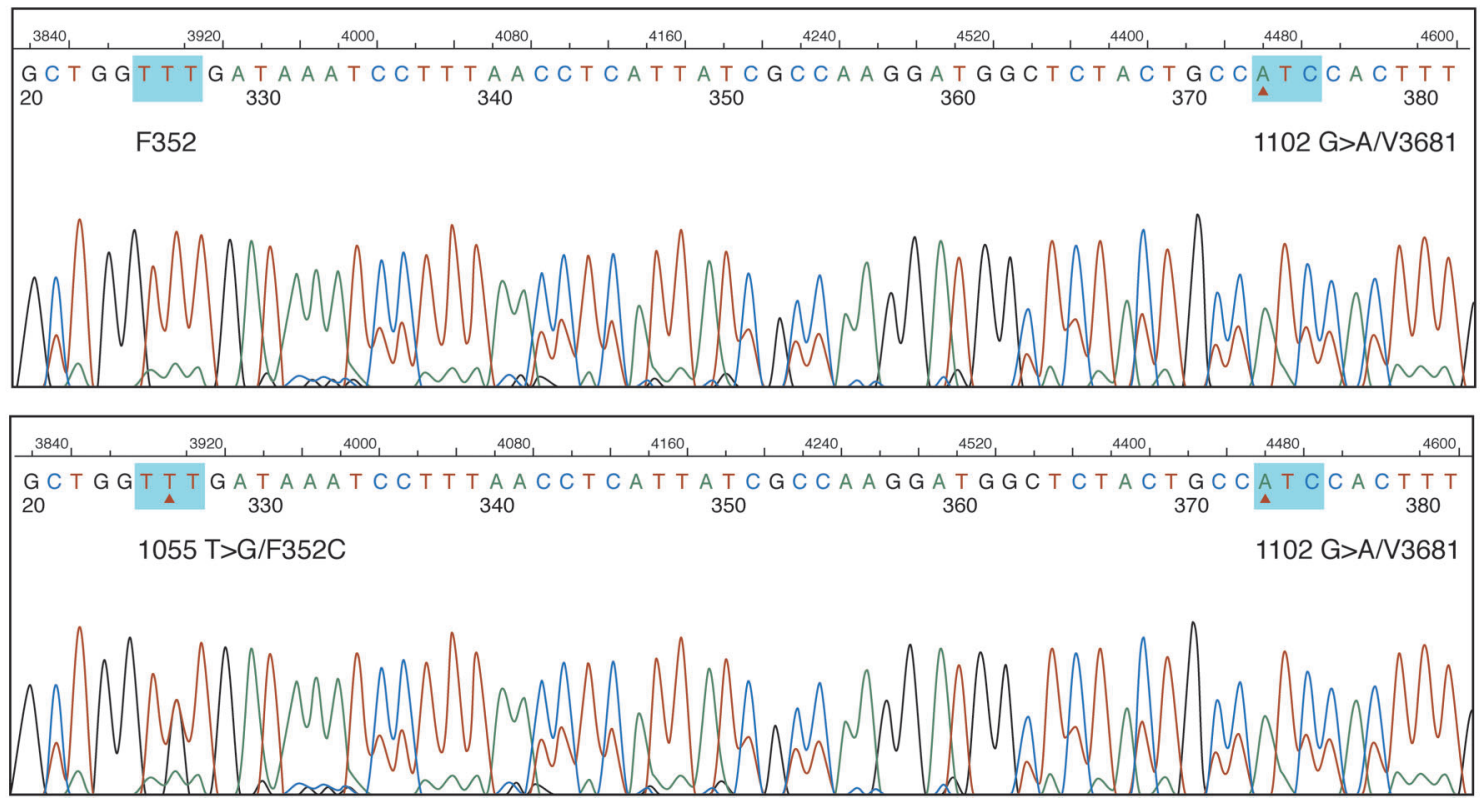

Patient 1

Patient 2

Fig. 3. CPT-II gene mutation and hepatic lipid accumulation. A. CPT-II gene exons $1-5$. B. Sequence fragments of the CPT-II gene exon 4 were amplified on the mitochondrial inner membrane. Mutation analysis was carried out using the specific primers to sequence the 1974 nucleotides encoding 658 amino acids. Comparison with the original sequence from GenBank identified two substitution sites: $1618(\mathrm{G} \rightarrow \mathrm{A})$ and $1858(\mathrm{~T} \rightarrow \mathrm{C})$, for V368I and F448L amino acid changes respectively.

confirmed in mouse models that presented with increased angiogenesis or progression from NASH to HCC. ${ }^{9,13}$

Adiponectin produced by adipose tissue is an antiinflammatory cytokine and regulates the metabolism of blood glucose and hepatic fatty acid; furthermore, adiponectin is decreased in patients with NAFLD. According to the data from animal models xenografted with HCC cell lines, this cytokine is able to increase JNK activation and induce cell apoptosis with AMPK alteration, which could also inhibit mTOR phosphorylation or xenograft growth in nude mice. ${ }^{12}$ Additionally, it has been shown to inhibit tumor growth and metastasis by suppression of tumor angiogenesis and of levels of circulating adiponectin in NASH model, which favours tumourigenesis. ${ }^{14}$
Furthermore, tumor necrosis factor (TNF) is a major adipose-derived cytokine and a potent activator of prooncogenic pathways involving mTOR, JNK, NF-кB and extracellular signal-regulated kinases (ERKs). Interleukin (IL)- 6 can bind to its receptors on liver or non-parenchymal cells to promote the binding of signal-transducing receptor (gp130) to the IL-6 receptor (R) complex to activate JAK1; the activation and phosphorylation of STAT3 factor then promotes cell proliferation and anti-apoptosis, ${ }^{12,15}$ indicating that the coefficient action of adiponectin from adipose tissues, along with related cytokines, affect fatty acid metabolism and hepatocyte malignant transformation via a multitude of signaling molecules or pathways. 


\section{Inducing roles of related proteins}

Sterol regulatory element binding proteins (SREBPS)

SREBPs are the main regulators of hepatic lipogenesis and activate hepatic steatosis at the early stage. In HCC, SREBP1 can significantly induce lipogenesis and its overexpression is associated with poor prognosis. ${ }^{9}$ SREBP1c mRNA has been reported as up-regulated in HCC tissues, as compared with adjacent non-cancerous or normal liver tissues. SREBP1 has also been shown to activate the fat pathway in human HCC cell lines. Moreover, inhibition of SREBP1 expression results in cell growth arrest and apoptosis, as well as increased cell proliferation ability. ${ }^{16}$

Tripartite motif 24 (TRIM24)

TRIM24 (previously known as TIF1 $\alpha$ ) is an epigenetic coregulator of some gene transcription that directly or indirectly inhibits mouse hepatic lipid accumulation, liver cell inflammation, liver fibrosis and hepatocyte damage. Additionally, global expression analyses of TRIM24 $4^{--}$livers unveiled signaling pathways (unfolded protein response or endoplasmic reticulum nuclear) that are closely associated with some features of patients with NAFLD, inflammatory stress, cell apoptosis and tissue damage. The loss of TRIM24 expression in liver tissues over time could lead to the progression from NAFLD to NASH or HCC in a time-dependent manner. ${ }^{17}$

\section{Osteopontin (OPN)}

Human hepatic OPN is a multifunctional protein involved in some pathological conditions including immunity, inflammation, fibrosis and the development of HCC in different tissues. Its deficiency in obese mice (fed with a high-fat diet) was shown to reduce hepatic steatosis and hepatocyte inflammation; in addition, these mice showed attenuated liver cell swelling, portal leukocyte infiltration and macrophage accumulation. OPN is induced by Hedgehog signaling, and may directly promote pro-fibrogenic responses in steatohepatitis; it also acts as a paracrine factor secreted by the bile duct or natural killer T (NKT) cells and as an autocrine factor promoting fibrosis in HSCs. Silencing of OPN gene transcription by specific shRNA leads to an increase in Bax expression, a decrease in protein expression of $\mathrm{BCl}-2 / \mathrm{BCl}-\mathrm{XL}$ and $\mathrm{X}$-linked inhibitor of apoptosis and in activation of NF-кB as well as induction of mitochondria-mediated apoptosis in the HCC cell line of LM3. ${ }^{18}$

\section{Promoting role of related cells}

\section{HSCs}

Human HSCs in the perisinusoidal space between sinusoids and hepatocytes are the predominant fibrogenic cells of liver tissues and are activated by hepatocyte injury, whereby they trans-differentiate from a quiescent state to proliferative matrix-producing myofibroblasts. The subsequent excessive production of extracellular matrix might result in liver cirrhosis. Human HSCs express amphiregulin and TNF- $\alpha$ converting enzyme (TCE) in a time-dependent manner. Human amphiregulin can increase cell proliferation through the EGFR, phosphatidylinositol-3 kinase (PI3K) and p38 mitogenic signaling pathways, and can also significantly induce up-regulation of fibrogenic biomarkers and the process of cell death. ${ }^{19}$ In addition, fatty liver represents a pro-metastatic microenvironment, with human HCC in the tissue promoting HCC migration and proliferation. ${ }^{20}$

\section{Immune cells}

Immune cells activated by metabolic stress will migrate to the liver, interact with cells in that tissue and drive the progression from NAFLD to HCC. For example, the metabolic activation of intrahepatic $\mathrm{CD}^{+}$T or NKT cells can promote NASH and HCC via crosstalk with liver cells. The NKT cells primarily cause steatosis in liver tissues by secreting a type II transmembrane protein (a TNF ligand super-family member, TNFSF14); moreover, it has been shown that both the $\mathrm{CD}^{+} \mathrm{T}$ and NKT cells cooperatively induce liver injury in a study of a new developed C57BL/ 6 mouse model that recapitulates the key features of metabolic syndrome by feeding a choline-deficient high-fat diet. ${ }^{21}$ Hepatic immune cells recognize cell injury or pathogen invasion via their intracellular or surface-expressed pattern recognition receptors, subsequently initiating signaling cascades that trigger the release of factors promoting inflammatory response during NAFLD progression; ${ }^{22}$ this phenomenon supports the theory that transition from NASH to HCC involves the liver cell lymphotoxin- $\beta$ receptor (LT $\beta R$ ) and NF- $\kappa B$ signaling.

\section{Polyploidization}

Polyploidization represents a huge change in the genome. During liver development-and even throughout adult lifephysiological polyploidization is likely to occur, but in NAFLD it takes place mainly due to cell cycle suspension in the S/G2 phases. Furthermore, hepatic oxidative stress promotes histopathological polyploidization, suggesting that it should be an early event in NAFLD that consequently contributes to HCC development. ${ }^{23}$

\section{Alterations of small molecules}

\section{Oxidative stress}

A serious imbalance was found between limited antioxidant defenses and excessive formation of reactive species produced by hepatic oxidative stress, such as the ROS or reactive nitrogen species (RNS). ${ }^{14}$ Obese persons can have increased free fatty acid (FFA) uptake, which in turn would stimulate FA oxidation to compensate for excessive liver fat storage and accelerate $\beta$-oxidation, leading to increased production of ROS that could damage the mitochondrial membrane and DNA.

Oxidative stress may also induce ER dysfunction that is associated with liver malignancy. The ER plays an important role in NAFLD pathogenesis; moreover, consecutive increasing oxidative stress, inflammation and activation of NF-кB and JNK signaling pathways lead to the accumulation of intracellular lipids. ${ }^{9} \mathrm{HCC}$ is characterized by high activation of ERK, which occurs via PIK13 activation. ER stress also activates the nuclear factor erythroid 2-related factor 2 and transcription factor-6, both of which regulate the small heterodimer partner nuclear receptor, leading to its suppression. This process, in turn, activates cyclin D1 and increases tumorigenicity and hepatocyte proliferation. ${ }^{24}$ 
Iron deposition

Liver is the body's main storage site for iron, due to its rich reticuloendothelial system. ${ }^{25}$ Excess liver iron may increase NASH risk and its progression to HCC. ${ }^{26,27}$ Abnormal iron deposition in liver is more frequent in NASH patients, and necroinflammation may be the driving factor. Iron and the coexistence of hyperinsulinemia are risk factors for NASH development and together they may contribute to insulin resistance, disease progression and HCC. Iron deprivation can suppress HCC growth, as shown in both in vivo and in vitro experiments. ${ }^{25,28}$

\section{Alcohol}

Recent data suggest that chronic alcohol intake results in the induction of hepatic cytochrome $\mathrm{P}_{450} 2 \mathrm{E} 1$ (CYP2E1), which leads to generation of ROS with direct and indirect carcinogenic consequences. ${ }^{29}$ Genetic factors regulating alcohol metabolism could predispose towards development of alcoholic pancreatitis. Studies have also revealed that alcohol can be metabolized by oxidative and non-oxidative pathways. The main oxidative pathways include those involving alcohol dehydrogenase $(A D H)$, aldehyde dehydrogenase $(A L D H)$ and CYP2E1. In addition, neurocan (expressed in neuronal tissue) is also expressed in liver, and the common polymorphism of its gene rs2228603 has been shown as associated with $\mathrm{HCC}$ in alcoholic liver disease. ${ }^{30}$

\section{MicroRNA (miR)}

miRs are highly conserved, small non-coding RNAs (about 18 25 nucleotides in length) that serve to regulate transcription or translation of target genes and fatty acid metabolism. Both miR-197 and miR-99 have been associated with liver fibrosis in NASH patients. Some studies have also identified miR-122 as a key regulator of glucose and lipid metabolism in liver tissues, ${ }^{31}$ and NAFLD patients have been shown to have significantly higher levels of circulating miR-122, miR-34a, and miR-16. During the development of NAFLD, patients with simple steatosis to steatohepatitis have shown that the serological levels of miR-122 and miR-34a are closely positively correlated with disease severity, liver enzyme activities, fibrosis stage and active inflammation; ${ }^{32}$ this finding suggests that alteration of circulating miR-122 could be an early event in the transition of nonalcoholic steatohepatitis to hepatocarcinogenesis. ${ }^{33}$

\section{Insulin resistance}

Hepatic steatosis is defined as lipid accumulation in hepatocytes and is very frequently found in adults and obese adolescents. Etiologically, obesity and its associated insulin resistance (IR) or excess alcohol intake are the most frequent causes of liver steatosis. Insulin, as a key hormone, regulates lipogenesis and lipolysis in adipose depots. Under IR conditions, the adipose tissue becomes resistant to the anti-lipolytic effect of insulin and fatty acid release becomes increased; ${ }^{34}$ this process is accompanied by increased lipolysis and/or fat intake, possibly promoting hepatic triglyceride synthesis and resulting in liver lipid accumulation. ${ }^{35,36}$

Lipid accumulation in liver tissues causes IR occurrence through the activation of the NF-кB pathway, leading to hyperinsulinemia and the activation of PI3K/Akt that may be a contributing factor to hepatocarcinogenesis. ${ }^{11}$ Hyperinsulinemia is characterized by up-regulated expression of the hepatic insulin-like growth factor-1 (IGF1), which subsequently stimulates cellular proliferation and inhibits cell apoptosis. Insulin also activates the insulin receptor substrate-1 (IRS-1), which has been shown as up-regulated in HCC. ${ }^{26}$ Indeed, IRS-1-mediated signals may act as survival factors, promoting hepatocyte proliferation via mitogen-activated protein kinase and PI3K, and protecting against transforming growth factor $\beta 1$ (TGF- $\beta 1$ )-induced apoptosis in HCC development. ${ }^{24}$

\section{Genetic factors}

Data from genome-wide association studies (GWAS) have shown that patatin-like phospholipase domain-containing protein 3 (PNPLA3) on chromosome 22 is involved in the metabolism of triglycerides and is a genetic factor that promotes NASH development. The single nucleotide polymorphism (SNP) rs738409 is closely related to fatty liver and involved in fibrosis progression of NAFLD. The $C<G$ variation in SNP rs738409 also increases risk of HCC in NAFLD patients, and it is expected to be related to $\mathrm{HCC}$ development in patients with NAFLD/NASH. ${ }^{37,38}$ Whole exome sequencing indicated that apolipoprotein B (APOB) mutations (c.6718A>T, K2240X) represent a paradigm of rare variant influence on liver fat content and HCC risk. Additionally, mutations in telomerase reverse transcriptase (TERT) have been associated with hepatic steatosis, and deficiency of TERT can reduce response to liver damage, thereby inducing the formation of steatosis and fibrosis. In conclusion, the occurrence of NAFLD-HCC seems to be influenced by common genetic variants, such as those in PNPLA3, and by rare genetic variants, such as those in the APOB and TERT genes. ${ }^{39}$

\section{Microbiota and toxic substances}

\section{Gut microorganisms}

In intestine, there are trillions of microorganisms including bacteria, archaea, yeasts and viruses that collectively represent the intestinal ecosystem that is involved in energy harvesting and fat storage. Several studies support the theory of a relationship existing between intestinal microbial changes and obesity and its related complications, including IR and NAFLD. Homoeostasis disorders between bacteria and host lead to increased intestinal permeability and production of pro-inflammatory molecules, endotoxemia and cytokines. The relationship between the gut microbiota and NAFLD is dependent upon altered metabolism of choline and bile acids and production of endogenous alcohol. It has also been implicated in the IR development that is involved in NAFLD pathogenesis via various mechanisms. The gut-liver axis is the way by which bacteria and their potential hepatotoxic products (lipopolysaccharides, DNA, RNA, etc.) can easily reach the liver. ${ }^{40,41}$

Primary bile acids, which are derived from cholesterol, become secondary bile acids via the action of intestinal microbes. Bile acids combing with farnesoid $X$ receptor (FXR) inhibits cholesterol $7 \alpha$-hydroxylase (CYP7A1) transcriptions to affect bile acid synthesis or SREBP-1c transcription, thereby reducing triglyceride synthesis and enhancing the PPAR $\alpha$ signaling to promote fatty acid $\beta$-oxidation. If the bile acids bind to the G-protein-coupled cell surface receptor 5 (TGR5), 
the result could be inhibition of inflammation through suppression of the NF-кB pathway in macrophages. ${ }^{12}$ Many genetic and environmental factors have been suggested to contribute to the development of obesity and NAFLD, but the exact mechanisms remain unknown and require further investigations.

Toxic substances

NAFLD has been implicated in some medical conditions, including IR, obesity, metabolic syndrome, hyperlipemia, hypertension, cardiovascular disease and diabetes. Glyceraldehydederived advanced glycation end-products (glycer-AGEs) are the predominant components of toxic AGEs (TAGE). Increasing data have suggested that TAGE, along with its cognate receptors, might change intracellular signaling and gene expression of pro-inflammatory molecules, and may also elicit generation of oxidative stress in liver cells such as the human HSCs. Circulating TAGE levels were shown to be significantly higher in NASH patients than in those with simple steatosis or in healthy subjects. Moreover, in the NASH patients, the TAGE levels inversely correlated with adiponectin. ${ }^{42}$

In addition, dietary or genetic obesity induces alterations of gut microbiota, thereby increasing the levels of deoxycholic acid (DCA), a gut bacterial metabolite known to cause DNA damage. Tumor cells often show higher rates of fatty acid synthesis and lipid accumulation; moreover, increased lipid availability in liver tissues might provide ATP and structural support for cancerous cell proliferation. The overexpression of key genes involved in regulation of lipogenesis correlates with tumor growth and poor prognosis of HCC. Hepatic lipid accumulation is accompanied by distinct patterns of perilipin expression. ${ }^{15}$ These findings suggest that abnormality of hepatic lipid accumulation might promote hepatocyte malignant transformation.

\section{NAFLD in hepatocarcinogenesis}

Liver derangements in lipid metabolism, importing FFAs and manufacturing, storing and exporting lipids could lead to NAFLD development. ${ }^{43}$ Although the majority of NAFLD patients presented with steatosis only, about $20 \%$ of patients present with NASH (as defined by microscopic findings and consisting of liver injury, steatosis, parenchymal and portal inflammation, and distinctive fibrosis). Dysregulation of the hormonal axes and cytokines in patients with NAFLD promotes a greater impairment of the cycle between metabolic and chronic inflammatory stimulus that might lead to malignant transformation of hepatocytes. ${ }^{44}$

In liver tissues, the DCA in the enterohepatic circulation provokes senescence-associated secretory phenotype human HSCs, which in turn secrete tumor-promoting factors, and thus facilitating HCC development (as evidenced in animal models following exposure to chemical carcinogen). ${ }^{45}$ High leptin level and low adiponectin level are hallmarks of obesity and involved in NAFLD pathogenesis and carcinogenesis. ${ }^{46}$ Obesity-promoted HCC has been shown to be dependent on increasing levels of IL-6 and TNF, which result in liver inflammation and oncogenic STAT3 activation. The long-term chronic inflammatory condition of obesity plus the higher levels of IL- 6 and TNF might represent a risk factor for HCC. ${ }^{47}$

A prospective study involving 25337 patients with HCC demonstrated that both excess body weight (SRR $=1.48,95 \%$ CI: $1.31-1.67)$ and obesity (SRR $=1.83,95 \% \mathrm{CI}: 1.59-2.11$ ) are related to increased risk of HCC occurrence, regardless of sex. ${ }^{48}$ Hepatic lipid accumulation promotes the release of proinflammatory cytokines and progression from steatosis to $\mathrm{NASH}$. Both diabetes and obesity lead to oxidative stress and lipid peroxidation of phospholipids, resulting in cell injury, necrosis or hepatocyte malignant transformation. ${ }^{49}$ Prospective studies with longer follow-up periods should be designed to screen NASH or NAFLD populations for specific biomarkers of malignant transformation of hepatocytes. ${ }^{50,51}$

\section{Perspectives}

In the past decade, the discussion around the substantial increase in NAFLD related to hypernutrition and HCC has become a cocktail party cliché, but its impact on public health cannot be dismissed. With our understanding of the relationships of both gradually deepening, we now see that NAFLD might promote the malignant transformation of hepatocytes as a result of liver lipid accumulation, hepatotoxicity, endoplasmic reticulum dysfunction, IR and abnormal fat metabolism. Although the exact mechanisms underlying the NAFLD tumor-promoting mechanisms triggered by hypernutrition remain to be explored, ${ }^{52}$ it is well recognized that those patients with excessive fat deposition are at greater risk of tumor-promoting inflammation conditions and should be treated in time to avoid hepatocyte malignant transformation.

\section{Acknowledgments}

This work was supported by grants from the Projects of the Priority Academic Program Development of Jiangsu Higher Education Institution (Qinglan Plan), Nantong Society Undertaking and Technological Innovation (HS2014078), National Natural Science Foundation (81200634 and 81370982) and the International S. \& T. Cooperation Program (2013DFA32150) of China.

\section{Conflict of interest}

None

\section{Author contributions}

Contributed equality to this work (JJG, MY, DBY), concept and literature search (JJG, LW), drafting the manuscript (JJG, MY, $\mathrm{XLY}, \mathrm{DBY}$ ), and critical revision for intellectual content (YDF).

\section{References}

[1] Ding J, Wang $\mathrm{H}$. Multiple interactive factors in hepatocarcinogenesis. Cancer Lett 2014;346:17-23, doi: 10.1016/j.canlet.2013.12.024.

[2] Hamid AS, Tesfamariam IG, Zhang Y, Zhang ZG. Aflatoxin B1-induced hepatocellular carcinoma in developing countries: Geographical distribution, mechanism of action and prevention. Oncol Lett 2013;5:1087-1092.

[3] Fan JG, Farrell GC. Epidemiology of non-alcoholic fatty liver disease in China. J Hepatol 2009;50:204-210. doi: 10.1016/j.jhep.2008.10.010.

[4] Wei Z, Doria C, Liu Y. Targeted therapies in the treatment of advanced hepatocellular carcinoma. Clin Med Insights Oncol 2013;7:87-102. doi: 10.4137/ CMO.S7633.

[5] Fan JG. Epidemiology of alcoholic and nonalcoholic fatty liver disease in China. J Gastroenterol Hepatol 2013;28 Suppl 1:11-17. doi: 10.1111/jgh. 12036.

[6] Yao D, Mizuguchi H, Yamaguchi M, Yamada H, Chida J, Shikata K, et al. Thermal instability of compound variants of carnitine palmitoyltransferase II and impaired mitochondrial fuel utilization in influenza-associated encephalopathy. Hum Mutat 2008;29:718-727. doi: 10.1002/humu.20717. 
[7] Yao M, Wang XY, Wang L, Yao DF, Yao DB. Antagonistic key molecule of mitochondrial carnitine shuttle system on effects of hepatic fat metabolism. J Nantong Uni (Med Sci) 2014;34:247-250. (in Chinese)

[8] Yao DB, Yao M, Wang L, Dong ZZ, Yao DF. Plasmid construction and variation analysis of exon-4 of carnitine palmitoyltransferase-II gene. J Clin Rehabil Tissue Engin Res 2011;15:9437-9440. (in Chinese)

[9] Stickel F, Hellerbrand C. Non-alcoholic fatty liver disease as a risk factor for hepatocellular carcinoma: mechanisms and implications. Gut 2010;59: 1303-1307. doi: 10.1136/gut.2009.199661.

[10] De Minicis S, Marzioni M, Benedetti A, Svegliati-Baroni G. New insights in hepatocellular carcinoma: from bench to bedside. Ann Transl Med 2013;1:15.

[11] Linhart KB, Glassen K, Peccerella T, Waldherr R, Linhart H, Bartsch $H$, et al. The generation of carcinogenic etheno-DNA adducts in the liver of patients with nonalcoholic fatty liver disease. Hepatobiliary Surg Nutr 2015;4:117-123.

[12] Jiang CM, Pu CW, Hou YH, Chen Z, Alanazy M, Hebbard L. Non alcoholic steatohepatitis a precursor for hepatocellular carcinoma development. World J Gastroenterol 2014;20:16464-16473. doi: 10.3748/wjg.v20.i44. 16464.

[13] Montella M, Crispo A, Giudice A. HCC, diet and metabolic factors: Diet and HCC. Hepat Mon 2011;11:159-162.

[14] Yoon HJ, Cha BS. Pathogenesis and therapeutic approaches for non-alcoholic fatty liver disease. World J Hepatol 2014;6:800-811. doi: 10.4254/wjh.v6. i11.800.

[15] Baffy G, Brunt EM, Caldwell SH. Hepatocellular carcinoma in nonalcoholic fatty liver disease: an emerging menace. J Hepatol 2012;56:1384-1391. doi: 10.1016/j.jhep.2011.10.027.

[16] Yamashita T, Honda M, Takatori H, Nishino R, Minato $H$, Takamura $H$, et al. Activation of lipogenic pathway correlates with cell proliferation and poor prognosis in hepatocellular carcinoma. J Hepatol 2009;50:100-110. doi: 10.1016/j.jhep.2008.07.036.

[17] Jiang S, Minter LC, Stratton SA, Yang P, Abbas HA, Akdemir ZC, et al. TRIM24 suppresses development of spontaneous hepatic lipid accumulation and hepatocellular carcinoma in mice. J Hepatol 2015;62:371-379. doi: 10. 1016/j.jhep.2014.09.026.

[18] Nagoshi S. Osteopontin: Versatile modulator of liver diseases. Hepatol Res 2014;44:22-30. doi: 10.1111/hepr.12166.

[19] McKee C, Sigala B, Soeda J, Mouralidarane A, Morgan M, Mazzoccoli G, et al. Amphiregulin activates human hepatic stellate cells and is upregulated in non alcoholic steatohepatitis. Sci Rep 2015;5:8812. doi: 10.1038/srep08812.

[20] Mikuriya $\mathrm{Y}$, Tashiro $\mathrm{H}$, Kobayashi $\mathrm{T}$, Kuroda $\mathrm{S}$, Abe $\mathrm{T}$, Hashimoto $\mathrm{M}$, et al. Clinicopathological features of hepatocellular carcinoma in patients with noncalcoholic fatty liver disease. Langenbecks Arch Surg 2015;400:471-476. doi: $10.1007 / \mathrm{s} 00423-015-1295-x$.

[21] Wolf MJ, Adili A, Piotrowitz K, Abdullah Z, Boege Y, Stemmer K, et al. Metabolic activation of intrahepatic CD8 + T cells and NKT cells causes nonalcoholic steatohepatitis and liver cancer via cross-talk with hepatocytes. Cancer Cell 2014;26:549-564. doi: 10.1016/j.ccell.2014.09.003.

[22] Bieghs $V$, Trautwein C. Innate immune signaling and gut-liver interactions in non-alcoholic fatty liver disease. Hepatobiliary Surg Nutr 2014;3:377-385.

[23] Gentric G, Maillet V, Paradis V, Couton D, L'Hermitte A, Panasyuk G, et al. Oxidative stress promotes pathologic polyploidization in nonalcoholic fatty liver disease. J Clin Invest 2015;125:981-992. doi: 10.1172/JCI73957.

[24] Noureddin M, Rinella ME. Nonalcoholic fatty liver disease, diabetes, obesity, and hepatocellular carcinoma. Clin Liver Dis 2015;19:361-379. doi: 10. 1016/j.cld.2015.01.012.

[25] Duan XY, Zhang L, Fan JG, Qiao L. NAFLD leads to liver cancer: Do we have sufficient evidence? Cancer Lett 2014;345:230-234. doi: 10.1016/j.canlet. 2013.07.033.

[26] Starley BQ, Calcagno CJ, Harrison SA. Nonalcoholic fatty liver disease and hepatocellular carcinoma: a weighty connection. Hepatology 2010;51: 1820-1832. doi: 10.1002/hep.23594.

[27] Sorrentino $P, D^{\prime}$ Angelo $S$, Ferbo $U$, Micheli $P$, Bracigliano $A$, Vecchione R. Liver iron excess in patients with hepatocellular carcinoma developed on non-alcoholic steato-hepatitis. J Hepatol 2009;50:351-357. doi: 10.1016/ j.jhep.2008.09.011.

[28] Ba Q, Hao M, Huang H, Hou J, Ge S, Zhang Z, et al. Iron deprivation suppresses hepatocellular carcinoma growth in experimental studies. Clin Cancer Res 2011;17:7625-7633. doi: 10.1158/1078-0432.CCR-10-3099.

[29] Kirstein MM, Vogel A. The pathogenesis of hepatocellular carcinoma. Dig Dis 2014;32:545-553. doi: 10.1159/000360499.

[30] Nischalke HD, Lutz P, Krämer B, Söhne J, Müller T, Rosendahl J, et al. A common polymorphism in the NCAN gene is associated with hepatocellular carcinoma in alcoholic liver disease. J Hepatol 2014;61:1073-1079. doi: 10. 1016/j.jhep.2014.06.006.

[31] Sun C, Fan JG, Qiao L. Potential epigenetic mechanism in non-alcoholic fatty liver disease. Int J Mol Sci 2015;16:5161-5179. doi: 10.3390/ ijms16035161.

[32] Cermelli S, Ruggieri A, Marrero JA, Ioannou GN, Beretta L. Circulating microRNAs in patients with chronic hepatitis $C$ and non-alcoholic fatty liver disease. PLoS One 2011;6:e23937. doi: 10.1371/journal.pone.0023937.

[33] Takaki Y, Saito Y, Takasugi A, Toshimitsu K, Yamada S, Muramatsu T, et al. Silencing of microRNA-122 is an early event during hepatocarcinogenesis from non-alcoholic steatohepatitis. Cancer Sci 2014;105:1254-1260. doi: 10.1111 /cas. 12498 .

[34] Frades I, Andreasson E, Mato JM, Alexandersson E, Matthiesen R, MartínezChantar ML. Integrative genomic signatures of hepatocellular carcinoma derived from nonalcoholic fatty liver disease. PLoS One 2015;10: e0124544. doi: 10.1371/journal.pone.0124544.

[35] Cohen JC, Horton JD, Hobbs HH. Human fatty liver disease: old questions and new insights. Science 2011;332:1519-1523. doi: 10.1126/science. 1204265.

[36] Gaggini M, Morelli M, Buzzigoli E, DeFronzo RA, Bugianesi E, Gastaldelli A. Non-alcoholic fatty liver disease (NAFLD) and its connection with insulin resistance, dyslipidemia, atherosclerosis and coronary heart disease. Nutrients 2013;5:1544-1560. doi: 10.3390/nu5051544.

[37] Oda K, Uto H, Mawatari S, Ido A. Clinical features of hepatocellular carcinoma associated with nonalcoholic fatty liver disease: a review of human studies. Clin J Gastroenterol 2015;8:1-9. doi: 10.1007/s12328-014-0548-5.

[38] Masarone M, Federico A, Abenavoli L, Loguercio C, Persico M. Non alcoholic fatty liver: epidemiology and natural history. Rev Recent Clin Trials 2014;9: 126-133. doi: 10.2174/1574887109666141216111143.

[39] Dongiovanni P, Romeo S, Valenti L. Hepatocellular carcinoma in nonalcoholic fatty liver: role of environmental and genetic factors. World J Gastroenterol 2014;20:12945-12955. doi: 10.3748/wjg.v20.i36.12945

[40] Alisi A, Ceccarelli S, Panera N, Nobili V. Causative role of gut microbiota in non-alcoholic fatty liver disease pathogenesis. Front Cell Infect Microbiol 2012;2:132. doi: 10.3389/fcimb.2012.00132.

[41] Gangarapu V, Yıldız K, Ince AT, Baysal B. Role of gut microbiota: obesity and NAFLD. Turk J Gastroenterol 2014;25:133-140. doi: 10.5152/tjg.2014. 7886.

[42] Takeuchi M, Sakasai-Sakai A, Takata T, Ueda T, Takino J, Tsutsumi M, et al. Serum levels of toxic AGEs (TAGE) may be a promising novel biomarker in development and progression of NASH. Med Hypotheses 2015;84:490-493. doi: 10.1016/j.mehy.2015.02.002.

[43] Berlanga A, Guiu-Jurado E, Porras JA, Auguet T. Molecular pathways in nonalcoholic fatty liver disease. Clin Exp Gastroenterol 2014;7:221-239.

[44] Streba LA, Vere CC, Rogoveanu I, Streba CT. Nonalcoholic fatty liver disease, metabolic risk factors, and hepatocellular carcinoma: an open question. World J Gastroenterol 2015;21:4103-4110. doi: 10.3748/wjg.v21.i14. 4103.

[45] Yoshimoto S, Loo TM, Atarashi K, Kanda H, Sato S, Oyadomari S, et al. Obesity-induced gut microbial metabolite promotes liver cancer through senescence secretome. Nature 2013;499:97-101. doi: 10.1038/nature12347.

[46] Marengo A, Rosso C, Bugianesi E. Liver cancer: connections with obesity, fatty liver, and cirrhosis. Annu Rev Med 2016;67:103-117. doi: 10.1146/ annurev-med-090514-013832.

[47] Park EJ, Lee JH, Yu GY, He G, Ali SR, Holzer RG, et al. Dietary and genetic obesity promote liver inflammation and tumorigenesis by enhancing IL- 6 and TNF expression. Cell 2010;140:197-208. doi: 10.1016/j.cell.2009.12.052.

[48] Chen Y, Wang X, Wang J, Yan Z, Luo J. Excess body weight and the risk of primary liver cancer: an updated meta-analysis of prospective studies. Eur J Cancer 2012;48:2137-2145. doi: 10.1016/j.ejca.2012.02.063.

[49] Kar P. Risk factors for hepatocellular carcinoma in India. J Clin Exp Hepatol 2014;4:S34-S42. doi: 10.1016/j.jceh.2014.02.155.

[50] Yao D, Jiang D, Huang Z, Lu J, Tao Q, Yu Z, et al. Abnormal expression of hepatoma specific gamma-glutamyl transferase and alteration of gammaglutamyl transferase gene methylation status in patients with hepatocellular carcinoma. Cancer 2000;88:761-769. doi: 10.1002/(SICI)1097-0142 (20000215)88:4<761::AID-CNCR5>3.0.CO;2-5.

[51] Raff EJ, Kakati D, Bloomer JR, Shoreibah M, Rasheed K, Singal AK. Diabetes mellitus predicts occurrence of cirrhosis and hepatocellular cancer in alcoholic liver and non-alcoholic fatty liver diseases. J Clin Transl Hepatol 2015;3: 9-16. doi: 10.14218/JCTH.2015.00001.

[52] Font-Burgada J, Sun B, Karin M. Obesity and cancer: the oil that feeds the flame. Cell Metab 2016;23:48-62. doi: 10.1016/j.cmet.2015.12.015. 